\title{
Critical Time to Nucleation: Graphite and Silicon Nanoparticle Generation by Laser Ablation
}

\author{
Sid Senadheera, ${ }^{1}$ Bo Tan, ${ }^{2}$ and Krishnan Venkatakrishnan ${ }^{1}$ \\ ${ }^{1}$ Department of Mechanical and Industrial Engineering, Ryerson University, 350 Victoria Street, Toronto, ON, Canada M2B $2 \mathrm{~K} 3$ \\ ${ }^{2}$ Department of Aerospace Engineering, Ryerson University, 350 Victoria Street, Toronto, ON, Canada M2B 2K3
}

Correspondence should be addressed to Krishnan Venkatakrishnan, venkat@ryerson.ca

Received 23 September 2008; Accepted 30 November 2008

Recommended by Apparao M. Rao

Femtosecond laser ablation hydrodynamics has been analyzed numerically. The nucleation process of two materials (silicon and graphite) in an expanding plume produced by a femtosecond laser ablation is analyzed within the framework of the ZeldovichRaizer theory (RZ theory). RZ theory is the most appropriate theory applicable to an expanding plume created by a femtosecond laser ablation and has been used to estimate the critical times in the evolution of nanoparticle formation. However, there is no experimental result to directly substantiate the theoretical model on a fundamental level. This paper gives an experimental approach to prove theoretical predictions of critical times to nucleation in laser-ablated graphite and silicon plumes. The critical time to nucleation deduced using the RZ theory was found to be in close agreement with pulse frequency at which fibrous nanoparticle aggregates start to form for both materials. We experimentally showed that the nanoparticle aggregates were produced consistently when the pulse frequency corresponding to the femtosecond laser time interval was shorter than the critical time to begin nucleation. Therefore the nucleation time acts as a threshold time length to begin nucleation.

Copyright (C) 2009 Sid Senadheera et al. This is an open access article distributed under the Creative Commons Attribution License, which permits unrestricted use, distribution, and reproduction in any medium, provided the original work is properly cited.

\section{Introduction}

Nanoparticles have attracted intensive interest in the nanotechnology applications due to their huge potentials in medicine and new engineering materials. Several methods have been developed to generate nanoparticles in large quantity, among which laser ablation is desired because of simplicity in configuration and short process time. Recently, femtosecond laser ablation has been investigated for nanoparticle generation and it was observed that the nanoparticle structure generated by femtosecond laser ablation presents unique characteristics, compared to nanoparticles that are generated with conventional long pulse lasers. The differences in ablation characteristics among femtosecond, picosecond, and nanosecond lasers are discussed in detail in [1].

Our recent research revealed that fibrous nanoparticle aggregates can be created by laser ablation of a target material in ambient atmosphere at room temperature [2]. To generate nanoparticle aggregates with high yield, laser pulses must be fed to the target material at a pulse frequency higher than the minimum value that was estimated through our experiments. A theoretical analysis using a derivation of the current hydrodynamic theory applied to laser-ablated silicon and carbon vapor plumes finds that the critical time to nucleation agrees well with the observed minimum pulse frequency at which aggregate formation begins.

\section{Experimental Methodology}

The experimental setup is shown schematically in Figure 1. We used a femtosecond laser capable of producing variable pulse widths and pulse frequencies. The laser source is an all-diode-pumped, direct-diode-pumped $\mathrm{Yb}$-doped fiber oscillator/amplifier system capable of producing variable pulse energies up to $10 \mathrm{~mJ}$ at a pulse frequency between $200 \mathrm{kHz}$ and $25 \mathrm{MHz}$. Average power varies between 0$20 \mathrm{~W}$. Arrays of microvias were drilled into a graphite target and a blank silicon wafer with laser beam at various pulse frequencies. The samples were then characterized using scanning electrical microscopy (SEM). Large amounts of fibrous nanostructures were observed surrounding the microvias, as shown in Figures 2 and 3. 


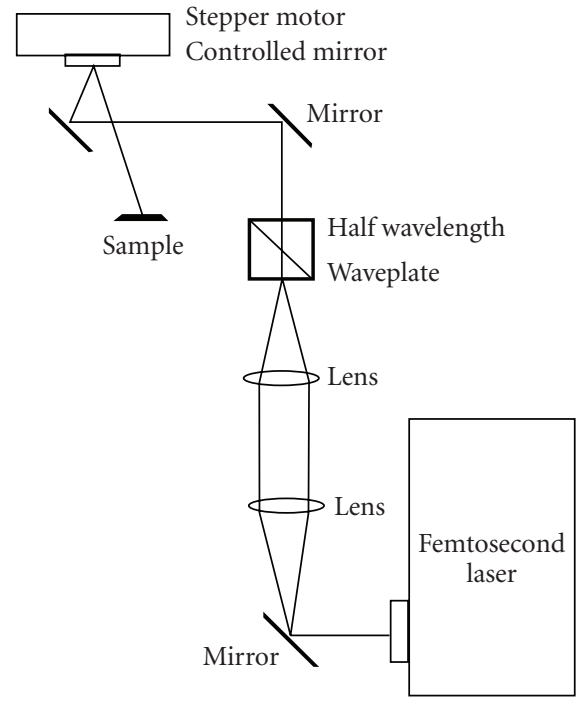

FIgURE 1: Setup for producing femtosecond laser ablation sample.

\section{Nanoparticle Production By Laser Ablation}

We observed that the formation of nanoparticle aggregates in silicon started at a pulse frequency of about $2 \mathrm{MHz}$. Until this pulse frequency, the formation of fibrous nanoparticle aggregates was not evident. At $2 \mathrm{MHz}$, aggregates are short and coexist with large amount of molten droplets. As the pulse frequency increases, the amount of molten droplets reduces and the aggregates grow longer, and finally form fibrous structures. For graphite, the formation of aggregates begins at $1 \mathrm{MHz}$. On the graphite target surface, the fibrous nanoparticle aggregates were denser at $1 \mathrm{MHz}$ than those on a silicon wafer surface at $2 \mathrm{MHz}$. The experimental results show that the minimum required pulse frequency to observe nanoparticle aggregates is material dependent.

To understand the formation of nanoparticle aggregates, it is important to understand how they evolve with time after the laser ablation. Characterizing our experimental results with an SEM, we observed that individual nanoparticles are bonded with each other to form nanoparticle aggregates, as shown in Figure 4.

As illustrated in Figure 5, during the laser ablation the material inside the plume evolves through several physical states before condensation. In the first step, the laser light is absorbed by free electrons due to inverse bremsstrahlung process in the semiconductor at the top layer. This is followed by a fast energy transfer to the lattice owing to electron-phonon coupling. After the energy transfer, a high temperature is reached in the lattice. The interaction of intense ultrashort light pulses with the target leads to a phase transformation (melting of surface, evaporation, and plasma formation). At first, the very high heating rates cause a rapid melting and the transition to an overcritical fluid. An overcritical fluid occurs when the material is in a nonequilibrium state of high pressure and high temperature. The rapidly increasing vapor pressure near the surface creates a large driving force for vapor expansion. The high temperature and density lead to pressures far exceeding the background gas pressure (of the order of 10-100 atm). The unusually high fluid pressure causes the plume front to expand very rapidly with time in a highly forward-directed pattern. At early times, the plume front is spherical in nature, but as time evolves the plume front becomes sharpened Figure 6. Along with sharpening, the expanding plume front splits into a fast and a slow moving clouds indicating plume splitting [3-6].

The expanding plume brings the hot-vaporized material from the target to a critical state by a process known as supercooling (cooling below the freezing point). Supercooling causes the vaporized material inside the plume to saturate and then form nuclei. The critical vapor within the plume (Figure 6) becomes supersaturated, leading to nanoparticle formation via condensation of nuclei. It has been shown by several researchers that nanoparticles bond together linearly and form similar structures to the fibers in nanoparticle aggregates [6] obtained by our experiments. The fundamental parts of the creation and evolution of the plume are shown in Figure 6. During condensation stage primarily formed nanoparticles may coalesce incompletely, which leads to the formation of aggregates [6], such as nanocrystal networks or nanofiber aggregates, and so on. Nanoparticle aggregates have the structure of web-like material shown in Figures 2, 3. At low pulse frequencies, in the range of $\mathrm{KHz}$, condensates have a structure of spherical nanoparticles, just after the condensation material that is still at an elevated temperature. The cooling process down to ambient temperature is very slow. This slow cooling process determines the physical structure of the condensates according to the pulse frequency that the target is subjected to.

\section{Analysis}

The first theoretical analysis of condensation dynamics in a rapidly expanding vapor was performed by Raizer et al. Anisimov et al. [7] and Anisimov and Luk'yanchuk [8] analyzed this theory with a modern perspective. Raizer considered the self-consistent problem of vapor condensation during expansion, as applied to the problem of cosmic dust in star formation regions. A constituent of the analysis is the classical theory of nucleation and production of nanoparticle aggregates which was developed in $[3,8]$. This theory of dynamic condensation of expanding vapor will be referred to as the Raizer-Zeldovich (RZ) theory, and is technically outlined in detail in [8]. An advantage of the RZ theory is that it is a clear manifestation of the physics of the process, and it also accounts correctly for the characteristic time scales for production and the dimensions of resulting clusters produced after the nucleation process. We use the RZ theory as a good theoretical model for estimating the time scales for nucleation which will be explained soon. The RZ theory has been applied (theoretically) to nanocluster production of silicon, germenium, and carbon using laser ablation in $[8,9]$.

During the phase transitional period, nucleation rate of particles ablated by the femtosecond laser is given by $v$. The time rate of change of nucleation is given by $d v / d t$. The 

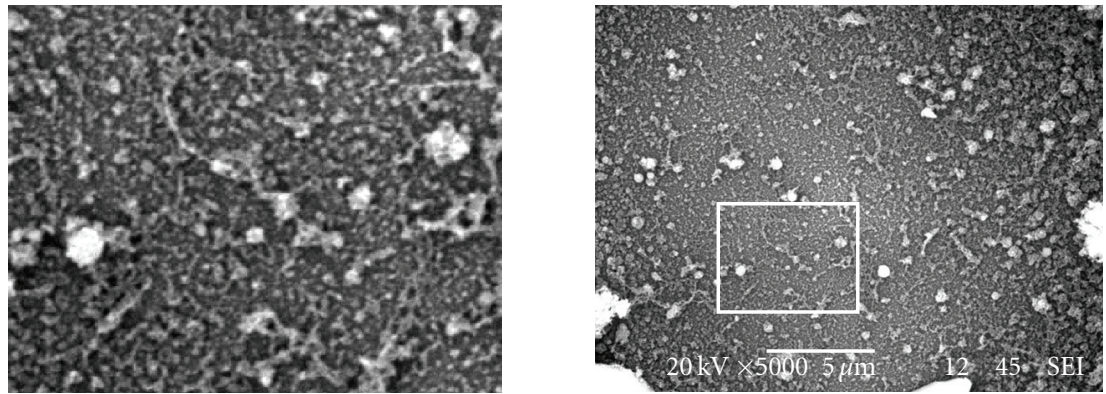

Silicon nanoparticle aggregates at $2 \mathrm{MHz}$

(a)
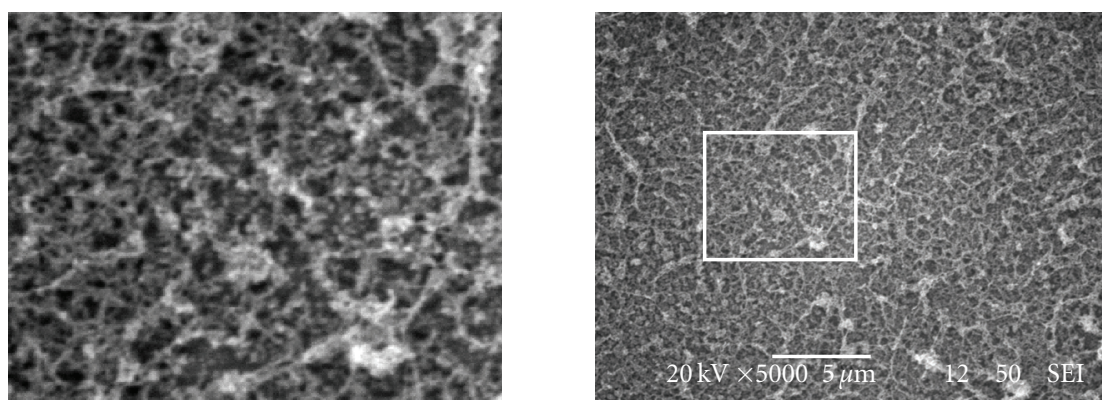

Silicon nanoparticle aggregates at $4 \mathrm{MHz}$

(b)
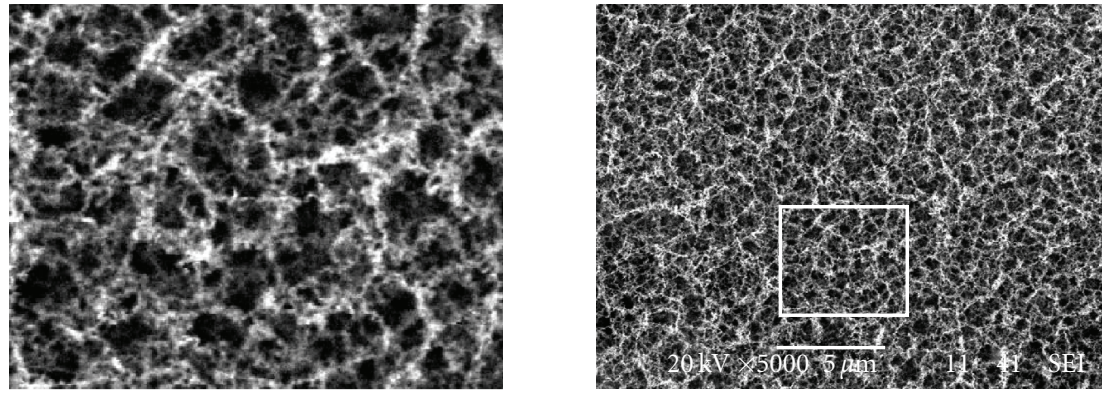

Silicon nanoparticle aggregates at $13 \mathrm{MHz}$

(c)

Figure 2: Silicon nanoparticle aggregate growth with laser pulse frequency.

nucleation process maximizes at $\left\{|\partial v / \partial t| \mid \mathrm{at}_{t=t_{e}}\right\}=0$. The instant of time $t_{e}$ is when the nucleation reaches maximum. The rate of formation of the nuclei is proportional to cluster growth which leads to nanoparticle formation within the advancing front of ablation laser plume Figure 6. According to Raizer-Zeldovich theory, the rate of nucleation can be written as

$$
\begin{aligned}
\frac{d v}{d t} & =K v(1-x)\left[\frac{\rho(t)}{\rho_{\text {start }}}\right] \cdot \exp \left[-\frac{T v}{T} \frac{1}{\theta^{2}}\right], \\
K v & =\sqrt{\frac{2 \sigma}{\pi m}} \cdot\left[\frac{\rho_{\text {start }}}{\rho_{L}}\right], \quad v=16 \pi \sigma^{3} \frac{M^{2}}{3 k_{B}^{3} q \rho_{L}}, \\
\theta & =\frac{\text { Teq }-T}{\text { Teq }}, \quad T_{v}=\frac{16 \pi \sigma^{3} M^{2}}{3 k_{B}^{3} q \rho_{L}} .
\end{aligned}
$$

To look at the formation of fibrous $3 \mathrm{D}$ nanoparticle aggregates, we have to look at the nucleation rate $d v / d t$. The behavior of the exponential factor of the nucleation rate is an extreme sharp function of supercooling [6] and is proportional to the term $\exp \left[-(T v / T)\left(1 / \theta^{2}\right)\right]$. Since we are only interested in finding the minimum repetition rate to form nanoparticle aggregates for graphite and silicon for beginning nucleation, we analyze only the time parameter of (1). Theoretically, we can see that the corresponding time where nucleation reaches it's maximum can be graphically represented (Figure 6 ). By separating the exponential part of the equation, we have

$$
\frac{d v}{d t} \propto \exp \left[-\frac{T v}{T} \frac{1}{\theta^{2}}\right]
$$



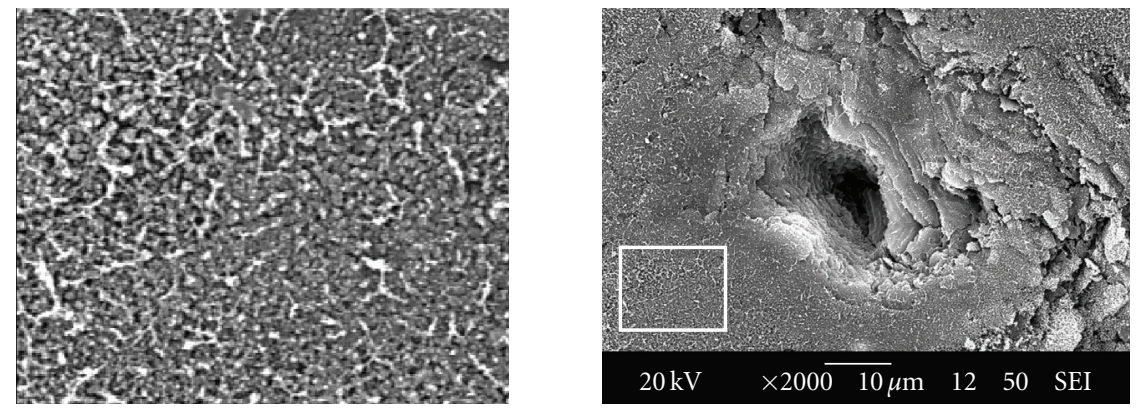

Graphite nanoparticle aggregates at $1 \mathrm{MHz}$

(a)
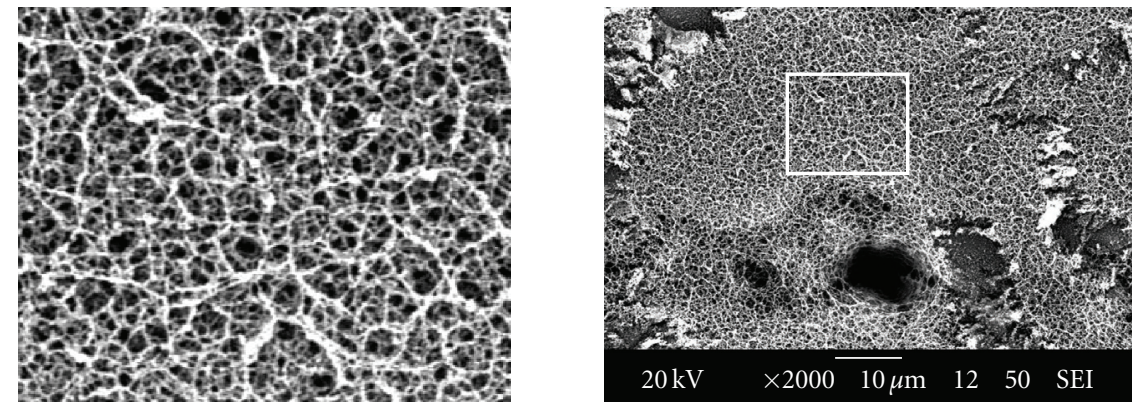

Graphite nanoparticle aggregates at $4 \mathrm{MHz}$

(b)
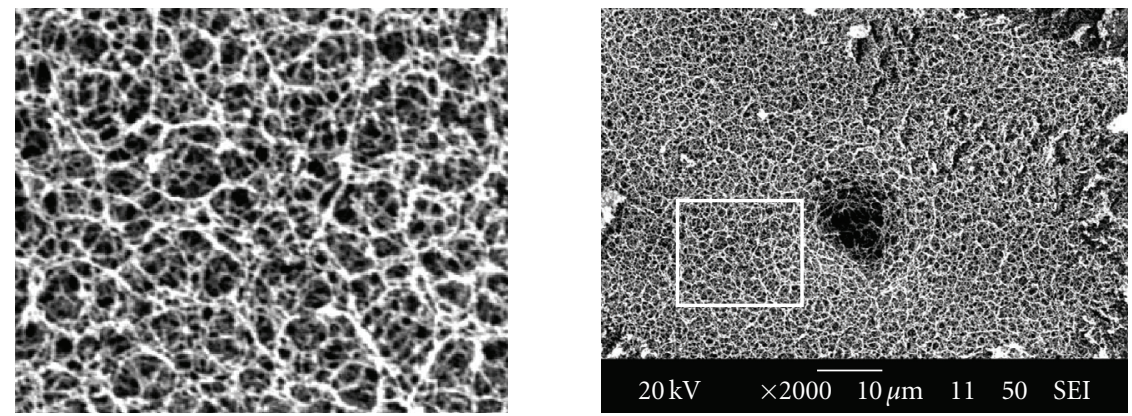

Graphite nanoparticle aggregates at $13 \mathrm{MHz}$

(c)

FIGURE 3: Graphite nanoparticle aggregate growth with laser pulse frequency.

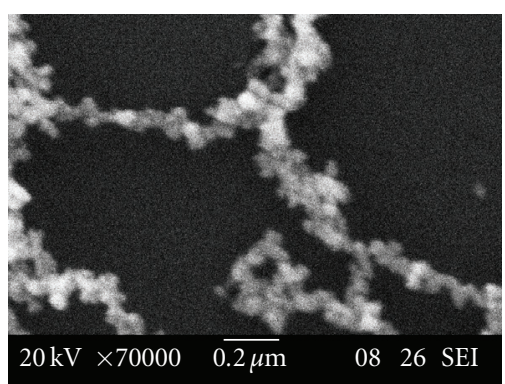

FIgURE 4: Nanoparticle aggregate chains.

\section{RZ Theory and the Nucleation Process}

There are few approaches to theoretically calculate the critical-time period toward nucleation. Out of these, we chose the classical hydrodynamics approach given by the RZ theory. The modern theories of nucleation represent only modifications of the classical theory, and they still remain unsuitable for quantitative prediction of experimental results. In order to create a universal description of supersaturated vapor nucleation, we need to get reliable theory such as the RZ theory that has been tested for half a century with various experiments. According to this theory, the nucleation rate during the critical time can be written as:

$$
\frac{d v}{d t}=\text { Const } \cdot \exp \left[-\frac{T v}{T} \frac{1}{\theta^{2}}\right]
$$

which can be numerically graphed as shown in Figure 7.

Figure 7 shows that nucleation starts just above 0.65 microsecond for silicon. This critical time to nucleation corresponds to a laser pulse frequency of $1.53 \mathrm{MHz}$, which 


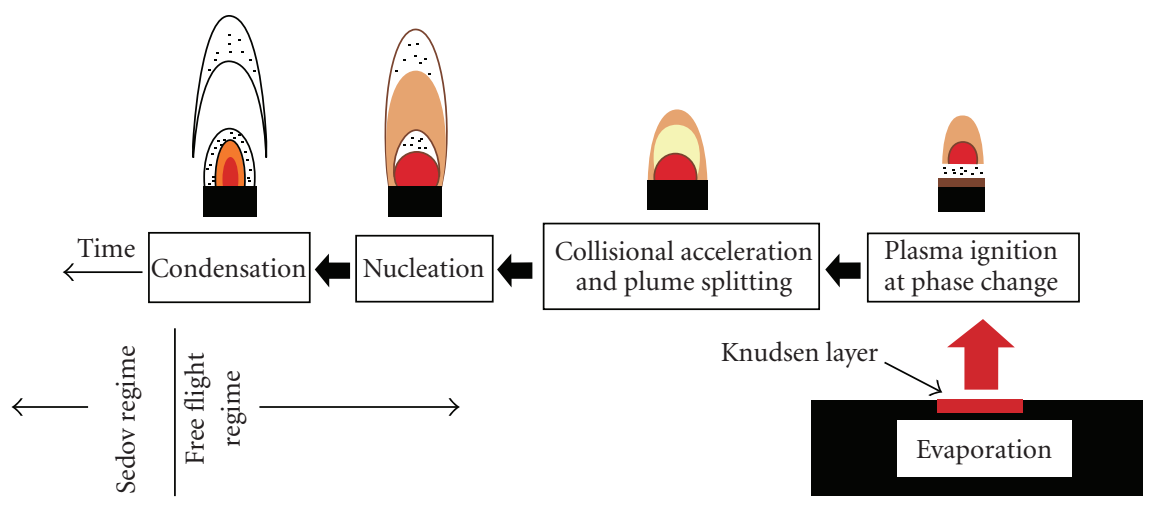

FIgURE 5: Time evolution of a femtosecond plume.

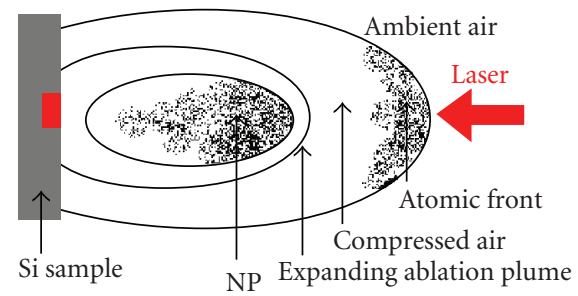

FIGURE 6: Constituents of the femtosecond plume.

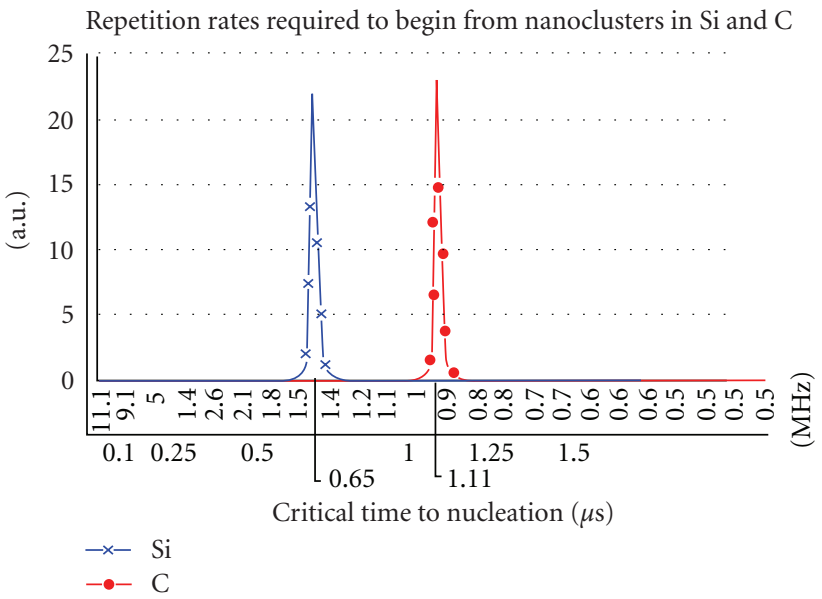

FIGURE 7: Critical time to nucleation in microsecond and repetition rate is in $\mathrm{MHz}$.

agrees well with our experimental observations where the silicon nanoparticle aggregates start to form at the pulse frequency of $2 \mathrm{MHz}$. The calculation also shows that graphite has a longer critical time of 1.11 microseconds, which is longer than that of silicon's. If the critical time to nucleation is the time to make minimum aggregations of the target material, pulse frequency at which graphite nanoparticles starts to aggregate should be lower than that of silicon's. Again, the experimental results on graphite target support this hypothesis and agree with the RZ theory.

Pulse frequency has a significant effect in creating nanofiber aggregates and keeping them continually forming.
For high pulse frequencies, ablation from consecutive pulses happens before the material on the surface of the target cools down. Therefore, the material that is ejected by a new pulse can have its ablated material condense (or bond) with the ablated material from the previous pulse which is still at a very high temperature. This is a possible explanation for how the nanoparticle structures are built dense and vertically in Figures 3, 4.

\section{Conclusion}

In our research, we measured the critical times required to form nanoparticle aggregates for graphite and silicon experimentally. We have elaborated how our experimental measurements for both materials agreed well with those predicted by RZ theory. Although the RZ theory has been previously supported experimentally by a few other researchers using particle sizes and atomic front velocity, and so on, there is no definitive evidence that gives strong experimental results to support the theory. One of the most important features of this theory is the ability to predict the critical time for nucleation. We showed how this important theoretical parameter (time for nucleation during phase transformation) agreed with experiment. Therefore, to the best of our knowledge, we have shown that Raizer-Zeldovich theory has been proven for the first time at it's most fundamental level in terms of time scale to a phase transition.

\section{Nomenclature}

$\rho_{L}$ : Density of liquid material

$M$ : Molecular weight

$x$ : Degree of condensation

$k_{B}$ : Boltzmann constant

$q$ : Heat of vaporization

$\sigma$ : Surface tension.

\section{Acknowledgment}

This research was funded by Natural Science and Engineering Research Council of Canada. 


\section{References}

[1] K. Venkatakrishnan and B. Tan, "Synthesis of fibrous nanostructures using ultrafast laser ablation under ambient condition and at mega hertz pulse frequency," Approved and pending publication in Optics Express.

[2] B. N. Chichkov, C. Momma, S. Nolte, F. von Alvensleben, and A. Tünnermann, "Femtosecond, picosecond and nanosecond laser ablation of solids," Applied Physics A, vol. 63, no. 2, pp. 109-115, 1996.

[3] R. Hergenröder, "A model for the generation of small particles in laser ablation ICP-MS," Journal of Analytical Atomic Spectrometry, vol. 21, no. 10, pp. 1016-1026, 2006.

[4] B. Rethfeld, V. V. Temnov, K. Sokolowski-Tinten, S. I. Anisimov, and D. von der Linde, "Dynamics of ultrashort pulselaser ablation: equation-of-state considerations," in High-Power Laser Ablation IV, vol. 4760 of Proceedings of SPIE, pp. 72-80, Taos, NM, USA, April 2002.

[5] A. Dalis and S. K. Friedlander, "Molecular dynamics simulations of the straining of nanoparticle chain aggregates: the case of copper," Nanotechnology, vol. 16, no. 7, pp. S626-S631, 2005.

[6] S. I. Anisimov and B. S. Luk'yanchuk, "Selected problems of laser ablation theory," Physics-Uspekhi, vol. 45, no. 3, pp. 293324, 2002.

[7] S. I. Anisimov, N. A. Inogamov, A. M. Oparin, et al., "Pulsed laser evaporation: equation-of-state effects," Applied Physics A, vol. 69, no. 6, pp. 617-620, 1999.

[8] B. S. Luk'yanchuk, W. Marine, S. I. Anisimov, and G. A. Simakina, "Condensation of vapor and nanoclusters formation within the vapor plume produced by nanosecond laser ablation of Si, Ge and C," Proc.SPIE, vol. 3618, pp. 434-452, 1999.

[9] L. J. Radziemski, R. W. Solarz, and J. A. Paisner, Laser Spectroscopy and Its Applications, CRC Press, Boca Raton, Fla, USA, 1987. 

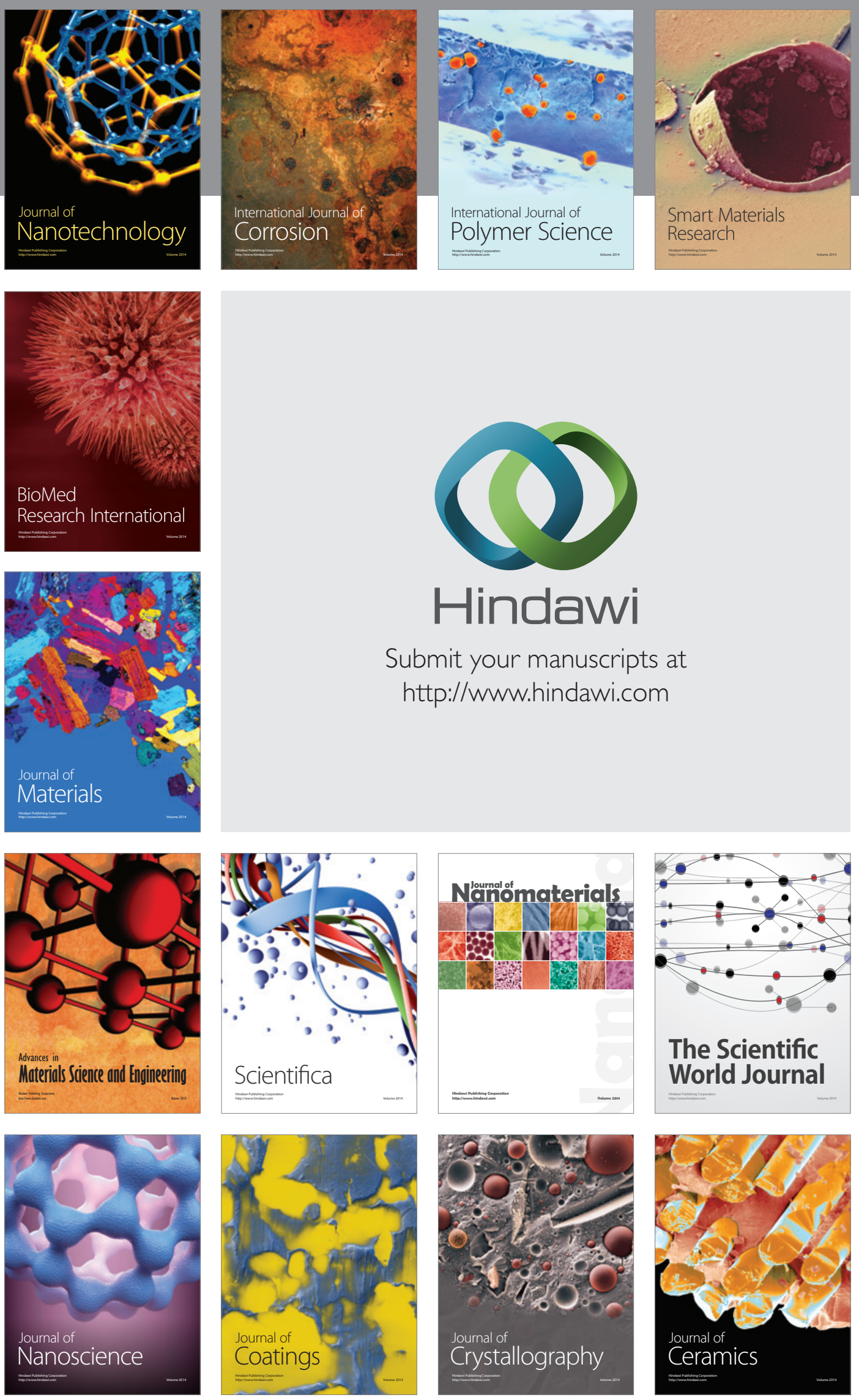

The Scientific World Journal

Submit your manuscripts at

http://www.hindawi.com

\section{World Journal}

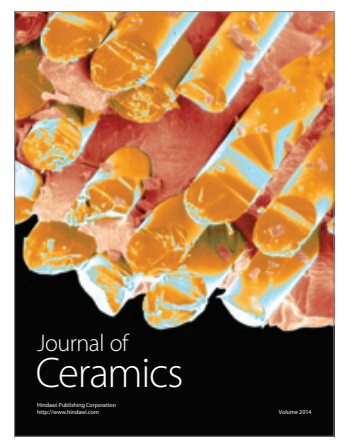

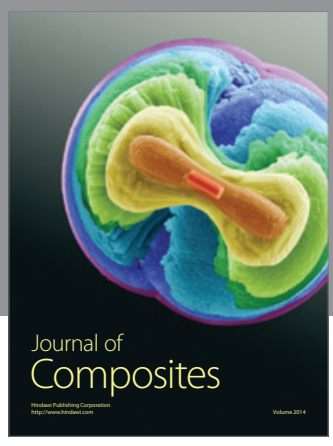
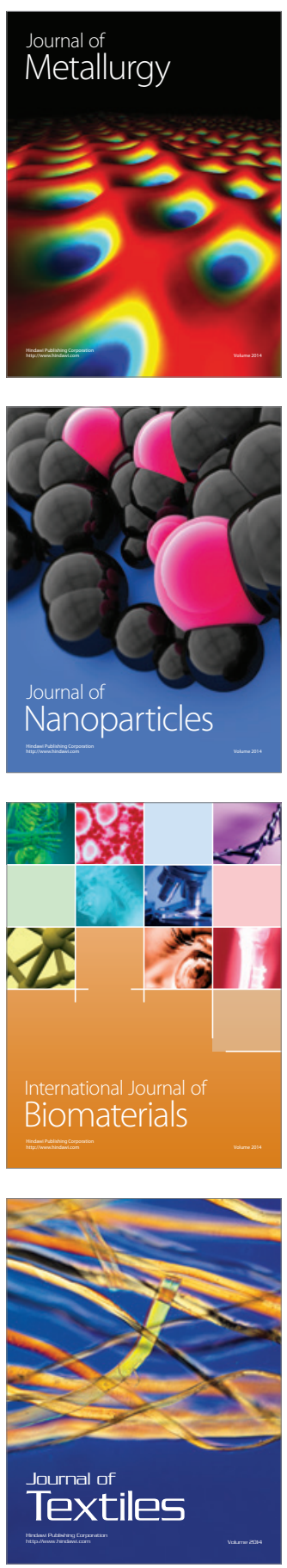